\title{
New 1-octanoyl-3-aryl thiourea derivatives: Solvent-free synthesis, characterization and multi-target biological activities
}

\author{
Fayaz Ali Larik, Aamer Saeed ${ }^{1}$, Pervaiz Ali Channar'1, Hammad Ismail², Erum Dilshad³ \\ and Bushra Mirza3
}

${ }^{1}$ Department of Chemistry, Quaid-i-Azam University, Islamabad 45320, Pakistan; ${ }^{2}$ Department of Biochemistry and Molecular Biology, University of Gujrat, Gujrat 50700, Pakistan; ${ }^{3}$ Department of Biochemistry, Quaid-i-Azam University, Islamabad 45320, Pakistan.

\begin{tabular}{|lr|}
\hline \multicolumn{2}{|l|}{ Article Info } \\
\hline Received: & 2 August 2016 \\
Accepted: & 11 October 2016 \\
Available Online: & 14 November 2016 \\
DOI: 10.3329/bjp.v11i4.29059 & \\
& \\
& \\
Cite this article: & \\
Saeed A, Larik FA, Channar PA, Is- \\
mail H, Dilshad E, and Mirza B. New \\
1-octanoyl-3-aryl thiourea derivatives: \\
solvent-free synthesis, characteriza- \\
tion and multi-target biological activi- \\
ties. Bangladesh J Pharmacol. 2016; 11: \\
894-902.
\end{tabular}

\begin{abstract}
An efficient solvent-free synthesis of a 10-member library of octanoyl linked substituted aryl thioureas was accomplished successfully. The octanoyl isothiocyanate was freshly prepared in excellent yield and purity by the reaction of potassium thiocyanate with octanoyl chloride followed by removal of potassium chloride by filtration. The reaction of the latter with a series of ten different substituted anilines by stirring at $60-65^{\circ} \mathrm{C}$ lead to the formation of the title compounds. The in vitro antifungal activity of newly synthesized compounds was evaluated against Aspergillus niger, A. flavus and Fusarium solani strains of pathogenic fungi. Antibacterial assay was carried out against Gram positive (Staphylococcus aureus, Micrococcus luteus) and Gram negative bacterial strains (Escherichia coli, Enterobacter aerogens). Furthermore, antioxidant potential and enzyme inhibition studies against a-amylase and butyryl cholinestrase were performed. The results obtained indicated moderate to excellent activities of most of the compounds whilst some derivatives showed potency higher than the standard used.
\end{abstract}

\section{Introduction}

Thioureas find extensive utility in synthetic, biological and commercial fields. It possesses a broad spectrum of biological activities including antibacterial, antifungal, anti-oxidant, and enzyme inhibition. Thioureas are the key precursors for the synthesis of a wide variety of heterocycles. In recent decades, several new methods have also been reported for the preparation of substituted thioureas. A mild and efficient microwave assisted synthesis of various di- and tri-substituted thioureas have been reported. Our research group has extensively been involved in the synthesis, biological assay and synthesis of heterocycles from thioureas (Saeed et al., 2015; Saeed et al., 2015; Saeed et al., 2014 and Saeed et al., 2016) their biological activities and theoretical studies have comprehensively been published (Saeed et al., 2015; Saeed et al., 2015; Saeed et al.,
2015; Saeed et al., 2014; Zaib et al., 2014; Saeed et al., 2013; Saeed et al., 2014; Saeed et al., 2010; Saeed et al., 2016; Saeed et al., 2009; Saeed et al., 2010 and Saeed et al., 2011). Thus, application of thioureas as excellent targets in medicinal chemistry has already been well established.

Herein, we report a clean, efficient and the solvent-free synthesis of a small library of 1-aroyl thioureas and their evaluation as antibacterial, antifungal, antioxidant and enzyme inhibitors.

\section{Materials and Methods}

\section{Experimental}

The mild solvent-free synthesis of a small library of ten candidates (3a-3j) was achieved by stirring freshly 


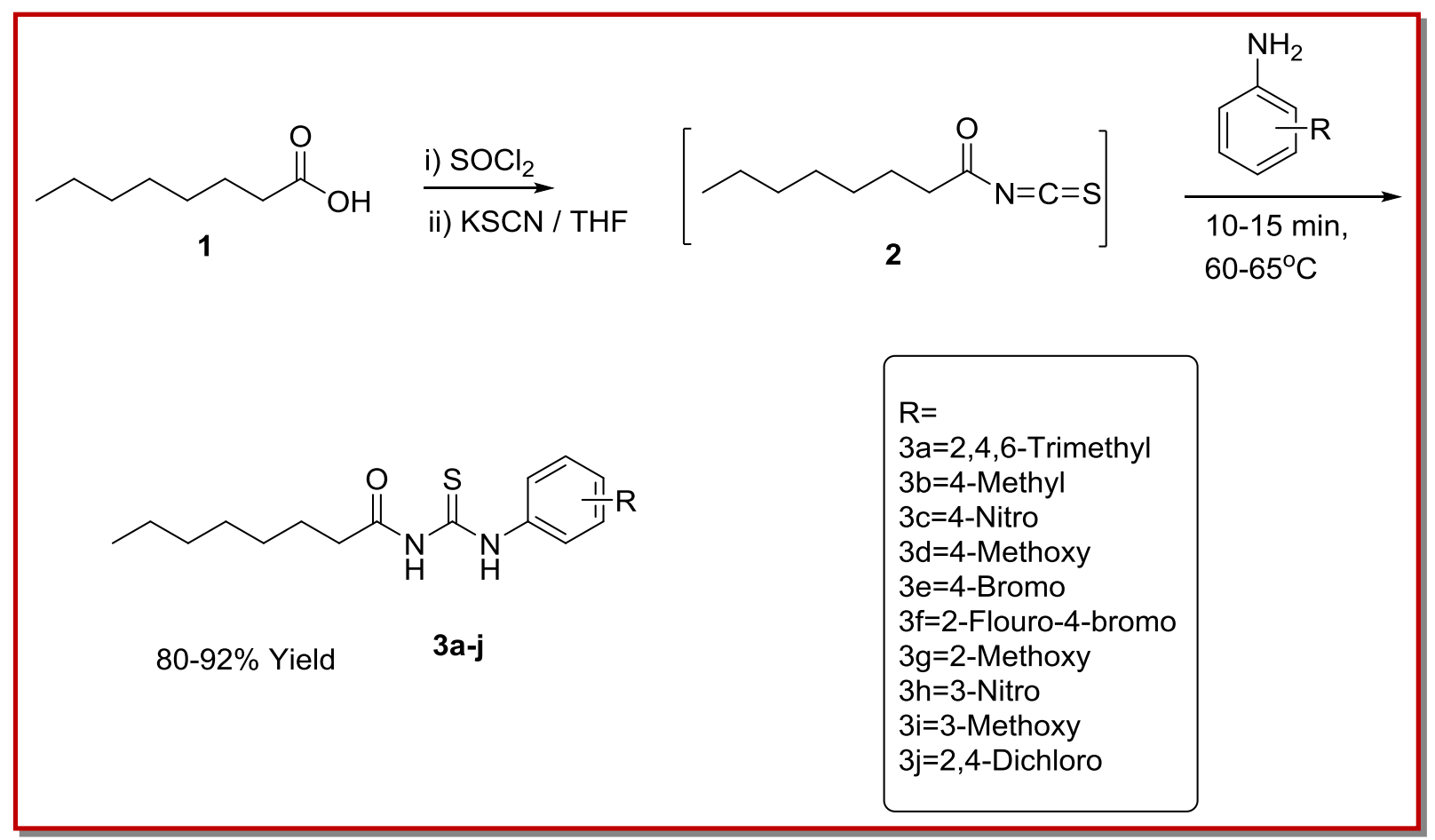

Scheme 1: Synthesis of substituted aryl thioureas

prepared octanoyl isothiocyanate with suitably substituted anilines. The complete description of the substituents for derivatives is enunciated in Scheme 1. This method provides facile access towards the synthesis of thiourea derivatives by employing solvent free conditions in a very short time. Moreover, the yield obtained by employing this methodology is excellent.

The melting points were determined on a Bio Cote SMP10-UK and are uncorrected. The chemicals like octanoic acid, ammonium thiocyanate, thionyl chloride, and aromatic amines, were purchased from SigmaAldrich and are used as received. The experiments were carried out in standard pyrex capable glassware chamber $(20 \mathrm{~mL})$. NMR spectra were recorded on a Bruker ARX, $300 \mathrm{MHz}$ spectrometer, ${ }^{1} \mathrm{H}$ NMR (300.13 MHz) and ${ }^{13} \mathrm{C}$ NMR (75.47 MHz) using internal standard $\mathrm{CDCl}_{3}$ solutions (7.28 ppm from TMS). The splitting of proton resonances in the reported ${ }^{1} \mathrm{H}$ NMR spectra are defined as s singlet, $\mathrm{d}$ doublet, $\mathrm{t}$ triplet, $\mathrm{q}$ quartet and $\mathrm{m}$ complex pattern; coupling constants were reported in $\mathrm{Hz}$. FT-IR spectra were recorded as $\mathrm{KBr}$ pellets on a Bio -Rad Excalibur FT-IR model FTS 3000 MX (400-4000 cm1) and the elemental analyses were performed using a LECO-932 CHNS analyzer.

\section{Antibacterial assays}

The antibacterial activity of the compounds was evaluated by disc diffusion assay as reported previously (Ellman et al., 1964). In experiment two Gram positive [Staphylococcus aureus (ATCC 6538) and Micrococcus luteus (ATCC 10240)] and two Gram negative [Escherichia coli (ATCC 15224) and Enterobacter aerogens
(ATCC 13048)] were cultured in nutrient broth for 24 hours at $37^{\circ} \mathrm{C}$. These cultured strains were used as inoculums $(1 \%)$ to run the assay. Each bacterial strain was added to the nutrient agar medium at $45^{\circ} \mathrm{C}$, poured into sterile petri plates and allowed to solidify. $5 \mu \mathrm{L}$ of the test compound with a final concentration of $200 \mu \mathrm{g} /$ $\mathrm{mL}$ was poured on sterile filter paper discs $(4 \mathrm{~mm})$ and placed on nutrient agar plates. Kanamycin and DMSO were used as positive- and-negative controls, respectively on each plate. The assay was performed in triplicate and the plates were incubated at $37^{\circ} \mathrm{C}$ for $24-$ 48 hours. The antibacterial activity of the compounds was determined by measuring the diameter of zones showing complete inhibition ( $\mathrm{mm}$ ) with the help of Vernier caliper.

\section{Antifungal assay}

Antifungal activity of synthesized compounds was measured by previously reported disc diffusion method (Mohammed et al., 2011) against Mucor species (FCBP 0300), Aspergillu sniger (FCBP 0198), Aspergillus flavus (FCBP 0064) and Fusarium solani (FCBP 0291). All fungal strains were cultured on Sabouraud dextrose agar (SDA) at $28^{\circ} \mathrm{C}$ for 5-7 days. Actively growing fungal spores of each strain were spread with the help of autoclaved cotton swaps on solidified SDA petri plates under sterile conditions. $5 \mu \mathrm{L}$ of each test compound with a final concentration of $200 \mu \mathrm{g} / \mathrm{mL}$ was poured on sterile filter paper discs $(4 \mathrm{~mm})$ and placed on SDA plates respectively. Terbinafine and DMSO served as positive and negative controls, respectively on each plate. All plates were incubated at $28^{\circ} \mathrm{C}$ for $5-7$ days and 
fungal growth was determined by measuring the growth diameter $(\mathrm{mm})$ with the help of a Vernier caliper.

\section{Anti-oxidant activity}

Radical scavenging activity of test compounds against stable free radical 2,2 diphenyl-1-picryl-hydrazyl (DPPH) was determined spectrophotometrically. Each test compound $(5 \mu \mathrm{L})$ with the final concentration of 200, 100 and $50 \mu \mathrm{g} / \mathrm{mL}$ was mixed with $100 \mu \mathrm{M} \mathrm{DPPH}$ $(95 \mu \mathrm{L})$ in 96-well microtiter plates. Ascorbic acid and DMSO were used as positive- and negative-control respectively. The experiment was performed in triplicate and reaction mixtures were incubated in dark for $30 \mathrm{~min}$ at $37^{\circ} \mathrm{C}$ in dark. After incubation, absorbance was measured at $515 \mathrm{~nm}$ by using microplate reader (BioTeK, Elx 800). IC 50 was calculated with Graph pad Prism 5.

\section{a-Amylase assay}

The compounds were tested for their enzyme inhibition activity against a-amylase by the previously reported method (Gorja et al., 2013). For assay $5 \mu \mathrm{L}$ of each test compound with the final concentration of 200, 100 and $50 \mu \mathrm{g} / \mathrm{mL}$ was mixed with $40 \mu \mathrm{L}$ of starch $(0.05 \%)$ and $30 \mu \mathrm{L}$ of potassium phosphate buffer ( $\mathrm{pH} 6.8$ ) in 96-well micro titer plates followed by the addition of $10 \mu \mathrm{L}$ of $\alpha-$ amylase enzyme (0.2 U/well). Acarbose and DMSO were used as positive- and negative-control respectively. The plates were incubated for $30 \mathrm{~min}$ at $50^{\circ} \mathrm{C}$ and $20 \mu \mathrm{L} \mathrm{HCl}(1 \mathrm{M})$ as stopping reagent was added. Then $100 \mu \mathrm{L}$ of iodine reagent $(5 \mathrm{mM}$ KI and 5 $\mathrm{mM} \mathrm{I}_{2}$ ) was added to check the presence and absence of starch and absorbance was measured at $540 \mathrm{~nm}$ with microplate reader (Bio Tek, Elx800). The experiments were performed in triplicate and $\mathrm{IC}_{50}$ was calculated with Graph pad Prism 5.

\section{Butyrylcholinesterase assay}

Ellman's method was used to determine the enzyme inhibition potential of the compounds against butyryl cholinestrase (BChE) (Ellman et al., 1966). In experiment butyrylthiocholine iodide (BChI) was used as substrates and assay was performed in triplicate in 96-well plates. The compound $(5 \mu \mathrm{L})$ with final concentration of 200, 100 and $50 \mu \mathrm{g} / \mathrm{mL}$ was mixed with $20 \mu \mathrm{L}$ of $100 \mu \mathrm{M}$ sodium phosphate buffer $(\mathrm{pH} 8.0)$ and $5 \mu \mathrm{L}$ BChE enzyme $(0.05 \mathrm{U} / \mathrm{mL})$. Then $10 \mu \mathrm{L}$ BChI $(4 \mathrm{mM})$ and 60 $\mu \mathrm{L}$ DTNB (3 $\mathrm{mM})$ was added. Galantamine hydrobromide (Sigma) and DMSO served as positiveand negative-controls respectively. The reaction mixtures were then incubated for $30 \mathrm{~min}$ at $37^{\circ} \mathrm{C}$. After incubation absorbance was measured at $405 \mathrm{~nm}$ using a microplate reader (Bio Tek Elx-800, USA) and $\mathrm{IC}_{50}$ was calculated by using Graph pad Prism 5.

\section{General procedure}

Freshly prepared octanoyl isothiocyanate was treated with respective aryl amines in a 1:1 Molar ratio under dry conditions. The reaction mixture was stirred at $60-$ $65^{\circ} \mathrm{C}$ for about $10-15 \mathrm{~min}$. On cooling, the reaction mixtures were slowly poured into acidified $(\mathrm{pH} 4-5)$ chilled water and stirred well $(50 \mathrm{~mL})$. The solid products obtained were separated by filtration and dried at room temperature.

\section{$\mathrm{N}$-(Mesitylcarbamothioyl)octanamide (3a)}

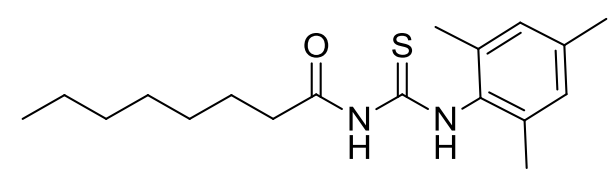

Light yellow solid; Yield: 91\%; Rf (n-hexane: Ethyl acetate $1: 1)$; 0.56 ; m.p: $195^{\circ} \mathrm{C}$; IR (neat,cm-1): 3219 (m, N$\mathrm{H}), 1680$ (s, v C=O), 1534 (v, d, NH), 1268 (v CNC). ${ }^{1} \mathrm{H}$ NMR (DMSO-d6), 300 MHz): 12.65 (s, 1H, NH), 12.03 (s, $1 \mathrm{H}, \mathrm{NH}), 7.48(\mathrm{~d}, 1 \mathrm{H}, J=8.3, \mathrm{Ar}-\mathrm{H}), 3.21(\mathrm{~s}, 6 \mathrm{H}, \mathrm{Ar}-\mathrm{H})$, 2.80 (s, 2H, Ar-H), 2.42 (t, 2H, J=3Hz), 1.93 (quint, 2H, $J=2 \mathrm{~Hz}$ ), 1.61 (quint, 2H), 1.32 (quint, 2H), 1.11 (quint, $2 \mathrm{H}), 0.90(\mathrm{sex}, 2 \mathrm{H}), 0.61(\mathrm{t}, 3 \mathrm{H}, J=1 \mathrm{~Hz}) ; 13 \mathrm{C}$ NMR (DMSO-d 6 );75.5 MHz): 179.6, 170.4, 162.19, 140.1, 133.4, 132.1, 130.3, 37.5, 32.3, 25.2, 22.1, 18.3, 15.4, 12.8, Anal. Calcd. For $\mathrm{C}_{18} \mathrm{H}_{28} \mathrm{~N}_{2} \mathrm{OS}$, C, 67.25; $\mathrm{H}, 8.81 ; \mathrm{N}, 8.71$; S 10.1; Found: C, 66.19; H, 8.60; N, 8.32; S, 9.74.

\section{$N$-(p-Tolylcarbamothioyl)octanamide (3b)}<smiles>CCCCCCCC(=O)NC(=S)Nc1ccc(C)cc1</smiles>

Light Pink solid; Yield: 88\%; Rf (n-hexane: Ethyl acetate 1:1); 0.50; m.p: $197^{\circ} \mathrm{C}$; IR (neat, $\left.\mathrm{cm}^{-1}\right): 3222$ (m, N-H), 1688 (s, v C=O), 1540 (v, d, NH), 1275 (v CNC). ${ }^{1} \mathrm{H}$ NMR (DMSO-d $), 300 \mathrm{MHz}): 12.61$ (s, 1H, NH), 11.89 (s, $1 \mathrm{H}, \mathrm{NH}), 7.5(\mathrm{dd}, 2 \mathrm{H}, J=8.3, J=8.0 \mathrm{~Hz}, \mathrm{Ar}-\mathrm{H}), 8.13$ (dd, $2 \mathrm{H}, J=7.0 \mathrm{~Hz}, \mathrm{~J}=6.7 \mathrm{~Hz}, \mathrm{Ar}-\mathrm{H}), 2.7$ (s, 2H, Ar-H), 2.2 (t, $2 \mathrm{H}, J=3 \mathrm{~Hz}$ ), 1.8 (quint, $2 \mathrm{H}, J=2 \mathrm{~Hz}$ ), 1.5 (quint, $2 \mathrm{H}$ ), 1.2 (quint, 2H), 1.0 (quint, $2 \mathrm{H}), 0.8(\mathrm{sex}, 2 \mathrm{H}), 0.5(\mathrm{t}, 3 \mathrm{H}$, $J=1 \mathrm{~Hz}) ;{ }^{13} \mathrm{C}$ NMR (DMSO-d 6 ); $75.5 \mathrm{MHz}$ ): 178.6, 173.4, 163.19, 141.1, 134.4, 132.5, 44.3, 372, 321, 256, 22.7, 15.2, 12.4, 10.2; Anal. Calcd. For $\mathrm{C}_{16} \mathrm{H}_{24} \mathrm{~N}_{2} \mathrm{OS}, \mathrm{C}, 65.71$; $\mathrm{H}, 8.27$; N, 8.27; S 10.96; Found: C, 64.19; H, 7.8; N, 7.75; S, 9.74.

N-((4-Nitrophenyl)carbamothioyl)octanamide (3c)<smiles>CCCCCCCC(=O)NC(=S)Nc1ccc([N+](=O)[O-])cc1</smiles>

Yellow solid; Yield: 80\%; Rf (n-hexane: Ethyl acetate 1:1); 0.61; m.p: $183^{\circ} \mathrm{C}$; IR (neat, $\mathrm{cm}^{-1}$ ): 3229 (m, N-H), 1685 (s, v, C=O), 1540 (v, d, NH), 1279 (v CNC). ${ }^{1} \mathrm{H}$ NMR (DMSO-d 6 ), $300 \mathrm{MHz}$ ): 12.65 (s, 1H, NH), 12.03 (s, $1 \mathrm{H}, \mathrm{NH}), 8.23(\mathrm{~d}, 1 \mathrm{H}, J=8.3, \mathrm{Ar}-\mathrm{H}), 7.83(\mathrm{~d}, 1 \mathrm{H}, J=8.03$, 
Ar-H), 2.5 (t, 2H, J=3Hz), 2.01 (quint, 2H, J=2Hz), 1.62 (quint, 2H), 1.42 (quint, 2H), 1.32 (quint,2H), 1.12 (sex, $2 \mathrm{H}), 0.81(\mathrm{t}, 3 \mathrm{H}, J=1 \mathrm{~Hz}) ;{ }^{13} \mathrm{C}$ NMR $\left(\mathrm{DMSO}_{-} \mathrm{d}_{6}\right) ; 75.5$ $\mathrm{MHz})$ : 180.6, 175.4, 165.19, 142.1, 135.4, 133.1, 131.4, 43.4., 39.3, 36.4, 24.5, 16.2, 14.5, Anal. Calcd. For $\mathrm{C}_{15} \mathrm{H}_{21} \mathrm{~N}_{3} \mathrm{O}_{3} \mathrm{~S}, \mathrm{C}, 55.71 ; \mathrm{H}, 6.51 ; \mathrm{N}, 12.71 ; \mathrm{S} 9.91$; Found: C, 54.19; H, 6.60; N, 11.32; S,8.74.

N-((4-Methoxyphenyl)carbamothioyl)octanamide (3d)<smiles>CCCCCCCC(=O)NC(=S)Nc1ccc(OC)cc1</smiles>

Light yellow solid; Yield: 95\%; Rf (n-hexane: Ethyl acetate 1:1); 0.61; m.p: $192^{\circ} \mathrm{C}$; IR (neat, $\left.\mathrm{cm}^{-1}\right)$ : 3234 (m, N$\mathrm{H}), 1671$ (s, v C=O), 1544 (v, d, NH), 1265 (v CNC). ${ }^{1} \mathrm{H}$ NMR (DMSO-d $), 300 \mathrm{MHz})$ : 12.31 (s, 1H, NH), 11.44 (s, $1 \mathrm{H}, \mathrm{NH}), 7.83(\mathrm{~d}, 1 \mathrm{H}, J=8.12, \mathrm{Ar}-\mathrm{H}), 8.13(\mathrm{~d}, 1 \mathrm{H}, J=$ 7.91, Ar-H), 3.98 (s, 3H), 2.42 (t, 2H, J=3Hz), 1.90 (quint, $2 \mathrm{H}, J=2 \mathrm{~Hz}$ ), 1.62 (quint, 2H), 1.32 (quint, 2H), 1.14 (quint, 2H), 0.9 (sex, 2H),0.62 (t, 3H, $J=1 \mathrm{~Hz}) ;{ }^{13} \mathrm{C}$ NMR (DMSO-d 6 ); 75.5 MHz): 179.6, 170.4, 162.19, 140.1, 133.4, 132.1, 54.6, 45.4, 40.2, 32.2, 25.4, 18.6, 12.3, 10.7 Anal. Calcd. For $\mathrm{C}_{16} \mathrm{H}_{24} \mathrm{~N}_{2} \mathrm{O}_{2} \mathrm{~S}, \mathrm{C}, 62.30 ; \mathrm{H}, 7.84 ; \mathrm{N}, 9.08 ; \mathrm{S}$ 10.40; Found: C, 62.19; H, 7.60; N, 8.32; S, 9.74.

N-((4-Bromophenyl)carbamothioyl)octanamide (3e)<smiles>CCCCCCCC(=O)NC(=S)Nc1ccc(Br)cc1</smiles>

Yellow solid; Yield: 98\%; Rf (n-hexane: Ethyl acetate 1:1); 0.51; m.p: $187^{\circ} \mathrm{C}$; IR (neat, $\mathrm{cm}^{-1}$ ): 3229 (m, N-H), 1685 (s, v, C=O), 1540 (v, d, NH), 1279 (v CNC). ${ }^{1} \mathrm{H}$ NMR (DMSO- $\mathrm{d}_{6}$ ), $\left.300 \mathrm{MHz}\right): 12.65$ (s, 1H, NH), 12.03 $(\mathrm{s}, 1 \mathrm{H}, \mathrm{NH}), 7.63(\mathrm{~d}, 1 \mathrm{H}, J=8.3, \mathrm{Ar}-\mathrm{H}), 7.65(\mathrm{~d}, 1 \mathrm{H}, J=$ 8.0, Ar-H), 2.4 (t, $2 \mathrm{H}, J=3 \mathrm{~Hz}), 2.1$ (quint, $2 \mathrm{H}, J=2 \mathrm{~Hz}$ ), 1.7 (quint, 2H), 1.5 (quint, 2H), 1.4 (quint, 2H), 1.2 (sex, $2 \mathrm{H}), 0.9$ (t, 3H, J=1Hz); ${ }^{13} \mathrm{C}$ NMR (DMSO-d $)$; $\left.75.5 \mathrm{MHz}\right)$ : 181.6, 174.4, 164.19, 143.1, 136.4, 134.1, 41.6, 37.2, 34.5, 30.4, 26.4, 23.7, 18.6, Anal. Calcd. For $\mathrm{C}_{15} \mathrm{H}_{21} \mathrm{BrN}_{2} \mathrm{OS}, \mathrm{C}$, 50.71; H, 5.92; N, 7.84; S 8.96; Found: C, 49.19; H, 6.60; $\mathrm{N}, 8.32 ; \mathrm{S}, 7.74$.

$N-((4-B r o m o-2-f l u o r o p h e n y l)$ carbamothioyl) octanamide (3f)<smiles>CCCCCCCC(=O)NC(=S)Nc1ccc(Br)cc1F</smiles>

White solid; Yield: $86 \%$; Rf (n-hexane: Ethyl acetate 1:1); 0.58; m.p: $201^{\circ} \mathrm{C}$; IR (neat, cm ${ }^{-1}$ ): 3230 (m, N-H), 1676 (s, v C=O), 1547 (v, d, NH), 1269 (v CNC). ${ }^{1} \mathrm{H}$ NMR
(DMSO-d $)$ ), $300 \mathrm{MHz}$ ): 12.11 (s, 1H, NH), 11.68 (s, 1H, $\mathrm{NH}), 7.93(\mathrm{~d}, 1 \mathrm{H}, J=7.10, \mathrm{Ar}-\mathrm{H}), 7.56(\mathrm{~d}, 1 \mathrm{H}, J=7.61$, Ar $-\mathrm{H}), 2.60(\mathrm{t}, 2 \mathrm{H}, J=3 \mathrm{~Hz}), 2.01$ (quint, $2 \mathrm{H}, J=2 \mathrm{~Hz}$ ), 1.82 (quint, 2H), 1.40 (quint, 2H), 1.32 (quint, 2H), 1.12 (sex, $2 \mathrm{H}), 0.61 \quad(\mathrm{t}, 3 \mathrm{H}, J=1 \mathrm{~Hz}) ;{ }^{13} \mathrm{C}$ NMR (DMSO-d $) ; 75.5$ MHz): 179.6, 170.4, 162.19, 140.1, 133.4, 132.1, 45.3, 40.5, 37.2, 32.4, 25.2, 22.4, 18.5, 15.5, 12.3, Anal. Calcd. For $\mathrm{C}_{15} \mathrm{H}_{20} \mathrm{BrFN}_{2} \mathrm{OS}, \mathrm{C}, 48.30 ; \mathrm{H}, 5.37 ; \mathrm{N}, 7.46$; S 8.54; Found: C, 47.19; H, 7.50; N, 7.21; S, 9.74.

N-((2-Methoxyphenyl)carbamothioyl)octanamide (3g)<smiles>CCCCCCCC(=O)NC(=S)Nc1ccccc1OC</smiles>

Black solid; Yield: 84\%; Rf (n-hexane: Ethyl acetate 1:1); 0.63; m.p: $210^{\circ} \mathrm{C}$; IR (neat, cm ${ }^{-1}$ ): 3245 (m, N-H), 1679 (s, v C=O), 1549 (v, d, NH), 1269 (v CNC). ${ }^{1} \mathrm{H}$ NMR (DMSO-d $), 300 \mathrm{MHz}): 11.14$ (s, 1H, NH), 11.68 (s, 1H, $\mathrm{NH}), 7.73(\mathrm{~d}, 1 \mathrm{H}, J=7.13, \mathrm{Ar}-\mathrm{H}), 7.56(\mathrm{~d}, 1 \mathrm{H}, J=7.45, \mathrm{Ar}$ $-\mathrm{H}), 7.06(\mathrm{~d}, 1 \mathrm{H}, J=7.13, \mathrm{Ar}-\mathrm{H}), 7.07$ (d, 1H, J = 7.45, Ar$\mathrm{H}), 3.45\left(\mathrm{~s}, 3 \mathrm{H}, \mathrm{OCH}_{3}\right), 2.6(\mathrm{t}, 2 \mathrm{H}, J=4 \mathrm{~Hz}), 2.0$ (quint, $2 \mathrm{H}, J=3 \mathrm{~Hz}$ ), 1.8 (quint, 2H), 1.4 (quint, 2H), 1.3 (quint, 2H), 1.1 (sex, 2H),0.6 (t, 3H, $J=1 \mathrm{~Hz}) ; 13 \mathrm{C}$ NMR (DMSO$\left.\mathrm{d}_{6}\right)$; $\left.75.5 \mathrm{MHz}\right)$ : 176.6, 171.4, 160.19, 141.1, 137.4, 135.4, 50,45, 41.6, 37.5, 32.4, 25, 22.3, 18.4, 15.8, 12.2, 14.1 Anal. Calcd. For $\mathrm{C}_{16} \mathrm{H}_{24} \mathrm{~N}_{2} \mathrm{O}_{2} \mathrm{~S}, \mathrm{C}, 62.30 ; \mathrm{H}, 7.84 ; \mathrm{N}, 9.08$; , 10.40 Found: C, 47.19; H, 7.50; N, 7.21; S, 9.74.

$\mathrm{N}$-((3-Nitrophenyl)carbamothioyl)octanamide (3h)<smiles>CCCCCCCC(=O)NC(=S)Nc1cccc([N+](=O)[O-])c1</smiles>

Yellow solid; Yield: 80\%; Rf (n-hexane: Ethyl acetate 1:1); 0.53; m.p: $170^{\circ} \mathrm{C}$; IR (neat, $\left.\mathrm{cm}^{-1}\right): 3229$ (m, N-H), 1670 (s, v C=O), 1543 (v, d, NH), 1260 (v, CNC). ${ }^{1} \mathrm{H}$ NMR (DMSO-d $), 300 \mathrm{MHz})$ : 12.22 (s, 1H, NH), 11.26 (s, $1 \mathrm{H}, \mathrm{NH}), 7.93(\mathrm{~d}, 1 \mathrm{H}, J=7.10, \operatorname{Ar}-\mathrm{H}), 7.56(\mathrm{~d}, 1 \mathrm{H}, J=$ 7.61, Ar-H), $7.92(\mathrm{~d}, 1 \mathrm{H}, J=7.10, \mathrm{Ar}-\mathrm{H}), 7.86(\mathrm{~d}, 1 \mathrm{H}, J=$ 7.61, Ar-H), $2.6(\mathrm{t}, 2 \mathrm{H}, J=3 \mathrm{~Hz}), 2.0$ (quint, $2 \mathrm{H}, J=2 \mathrm{~Hz}$ ), 1.8 (quint, 2H), 1.4 (quint, 2H), 1.3 (quint,2H), 1.1 (sex, 2H), $0.6(\mathrm{t}, 3 \mathrm{H}, \mathrm{J}=1 \mathrm{~Hz}) ;{ }^{13} \mathrm{C}$ NMR (DMSO-d $)$; $75.5 \mathrm{MHz}$ ): 179.6, 170.4, 162.19, 140.1, 133.4, 132.1, 46.4, 40.7, 37.3, 32.4., 25.8, 22.6, 18.6, 15.4, 12.8, Anal. Calcd. For $\mathrm{C}_{15} \mathrm{H}_{2}$ $\mathrm{N}_{3} \mathrm{O}_{3} \mathrm{~S}, \mathrm{C}, 55.71 ; \mathrm{H}, 6.54 ; \mathrm{N}, 12.99 ; \mathrm{S}$ 9.91; Found: C, $54.19 ; \mathrm{H}, 6.40 ; \mathrm{N}, 11.21 ; \mathrm{S}, 8.74$.

N-((3-Methoxyphenyl)carbamothioyl)octanamide (3i)<smiles>CCCCCCCC(=O)NC(=S)Nc1cccc(OC)c1</smiles> 
Yellow solid; Yield: 88\%; Rf (n-hexane: Ethyl acetate 1:1); 0.51; m.p: $187^{\circ} \mathrm{C}$; IR (neat, $\mathrm{cm}^{-1}$ ): 3226 (m, N-H), 1680 (s, v, C=O), 1535 (v, d, NH), 1270 (v CNC). ${ }^{1} \mathrm{H}$ NMR (DMSO-d $), 300 \mathrm{MHz})$ : 12.12 (s, 1H, NH), 11.76 (s, $1 \mathrm{H}, \mathrm{NH}), 7.23$ (dd, 1H, J = 8.3, J=7.2Hz, Ar-H), 7.83 (dd, $1 \mathrm{H}, J=8.0, J=7.4 \mathrm{~Hz}, \mathrm{Ar}-\mathrm{H}), 7.43(\mathrm{dd}, 1 \mathrm{H}, \mathrm{J}=7.12 \mathrm{~Hz})$, $7.41(\mathrm{dd}, 1 \mathrm{H}, \mathrm{J}=7.12 \mathrm{~Hz}), 3.7\left(\mathrm{~s}, 3 \mathrm{H}, \mathrm{OCH}_{3}\right), 2.5(\mathrm{t}, 2 \mathrm{H}$, $J=4 \mathrm{~Hz}$ ), 2.0 (quint, $2 \mathrm{H}, J=3 \mathrm{~Hz}$ ), 1.6 (quint, $2 \mathrm{H}$ ), 1.4 (quint, 2H), 1.3 (quint, 2H), 1.1 (sex, $2 \mathrm{H}), 0.8(\mathrm{t}, 3 \mathrm{H}$, $J=1 \mathrm{~Hz}$ ); ${ }^{13} \mathrm{C}$ NMR (DMSO-d 6 75.5 MHz): 179.21, 174.4, 165.19, 142.1, 135.4, 133.1, 56,43, 39.8, 36.9, 30.6, 24.8, 21.5, 16.8, 14.7, 12.8, 9.1; Anal. Calcd. For $\mathrm{C}_{15} \mathrm{H}_{21} \mathrm{~N}_{3} \mathrm{O}_{3} \mathrm{~S}$, C, 55.71; H, 6.51; N, 12.71; S 9.91; Found: C, 54.19; H, $6.60 ; \mathrm{N}, 11.32 ; \mathrm{S}, 8.74$.

\section{$\mathrm{N}$-((2,4-Dichlorophenyl)carbamothioyl)octanamide} (3j)<smiles>CCCCCCCC(=O)NC(=S)Nc1ccc(Cl)cc1Cl</smiles>

White solid; Yield: 86\%; Rf (n-hexane: Ethyl acetate 1:1); 0.49; m.p: $198^{\circ} \mathrm{C}$; IR (neat, cm-1): 3234 (m, N-H), 1670 (s, v C=O), 1549 (v, d, NH), 1261 (v CNC). ${ }^{1} \mathrm{H}$ NMR (DMSO-d 6 ), $300 \mathrm{MHz}$ ): 12.25 (s, 1H, NH), 11.63 (s, 1H, $\mathrm{NH}), 7.83(\mathrm{~d}, 1 \mathrm{H}, J=7.12$, Ar-H), $7.53(\mathrm{~d}, 1 \mathrm{H}, J=7.52, \mathrm{Ar}$ $-\mathrm{H}), 7.52(\mathrm{~d}, 1 \mathrm{H}, J=7.52, \mathrm{Ar}-\mathrm{H}), 2.8(\mathrm{t}, 2 \mathrm{H}, J=3 \mathrm{~Hz}), 2.11$ (quint, $2 \mathrm{H}, J=2 \mathrm{~Hz}$ ), 1.93 (quint, $2 \mathrm{H}$ ), 1.32 (quint, 2H), 1.1 (quint,2H), 0.92 (sex, 2H),0.86 (t, 3H, $J=1 \mathrm{~Hz}) ; 13 \mathrm{C}$ NMR (DMSO-d 6 ); $75.5 \mathrm{MHz}$ ): 178.6, 173.4, 164.19, 142.1, 135.2, 131.7, 42.9, 39.5, 35.7, 33.9, 27.8, 24.9, 19.7, 16.5, 14.9, Anal. Calcd. For $\mathrm{C}_{15} \mathrm{H}_{20} \mathrm{BrFN}_{2} \mathrm{OS}, \mathrm{C}, 51.30 ; \mathrm{H}, 5.80 ; \mathrm{N}$, 8.06; S 9.24; Found: C, 50.19; H, 5.50; N, 7.21; S, 8.74.

\section{Results}

\section{Antibacterial assay}

The synthesized compounds were evaluated for their potential antibacterial activity by using four different bacterial strains (Table I). The results showed that two compounds $3 a$ and $3 c$ showed antibacterial activity against all the tested bacterial strains which represent that these compounds are equally activity against Gram positive as well as Gram negative bacteria. The compound $3 \mathbf{i}$ exhibited antibacterial good activity against three bacterial strains except Enterobacter aerogenes. The compounds $3 \mathrm{~g}$ and $3 \mathrm{~h}$ showed antibacterial activity only against Staphylococcus aureus. Overall a range of antibacterial activity has been exhibited by the tested compounds.

\section{Antifungal assay}

All the tested compounds (3a-3j) showed significant antifungal activity against all tested fungal strains indicating that these compounds are broad spectrum antifungal candidates (Table II). The highest activity was measured against Aspergillus flavus and Aspergillus niger. These are very interesting findings that some compounds, e.g $\mathbf{3 a}$ and $\mathbf{3}$ c possess both antibacterial as well as significant antifungal activity against all tested strains.

\section{Butyryl cholinesterase assay and anti-oxidant assay}

The compounds were screened for their radical scavenging activity through DPPH assay (Table III). The results showed that $\mathbf{3 a}, \mathbf{3 b}, \mathbf{3 c}$ and $\mathbf{3 d}$ were found anti-oxidant and the rest of the compounds did not show any anti-oxidant activity.

\section{a-Amylase assay}

The compounds were evaluated for their enzyme inhibition potential against a-amylase enzyme and results in the form of $\mathrm{IC}_{50}$ values are given in Table III. Experiment was performed in triplicates and acarbose ( $\mathrm{IC}_{50} 17.1 \mu \mathrm{g} / \mathrm{mL}$ ) was used as positive control. The results showed that the compounds $3 \mathrm{f}, 3 \mathrm{~g}$ and $3 \mathrm{~h}$ exhibited enzyme inhibition activity with $\mathrm{IC}_{50}$ values of 282.1, 294.2 and $285.1 \mu \mathrm{g} / \mathrm{mL}$ respectively.

\section{Table I}

Antibacterial activity of the compounds

\begin{tabular}{|l|ccccc|}
\hline \multirow{2}{*}{ SL. No. } & Compound code & \multicolumn{4}{c|}{ Zones of inhibition $(\mathrm{mm})$ at $200 \mu \mathrm{g} / \mathrm{mL}$} \\
\cline { 3 - 6 } & & Micrococcus luteus & Staphylococcus aureus & Escherichia coli & Enterobacter aerogenes \\
1 & $3 \mathrm{a}$ & 25 & 27 & 26 & 25 \\
2 & $3 \mathrm{~b}$ & 27 & 28 & 30 & 25 \\
3 & $3 \mathrm{c}$ & 30 & 32 & 29 & 26 \\
4 & $3 \mathrm{~d}$ & 29 & 28 & 30 & 22 \\
5 & $3 \mathrm{e}$ & 27 & 19 & 22 & 19 \\
6 & $3 \mathrm{f}$ & 25 & 17 & 24 & 20 \\
7 & $3 \mathrm{~g}$ & 20 & 16 & 26 & 20 \\
8 & $3 \mathrm{~h}$ & 23 & 17 & 20 & 10 \\
9 & $3 \mathrm{i}$ & 18 & 18 & 16 & 20 \\
10 & $3 \mathrm{j}$ & 12 & 13 & 17 & 15 \\
11 & Kanamycin & 32 & 34 & 28 & 24 \\
\hline
\end{tabular}


Table II

\section{Antifungal activity of the compounds}

\begin{tabular}{|l|ccccc|}
\hline \multirow{2}{*}{ SL. No. } & Compound code & \multicolumn{4}{c|}{ Zones of inhibition $(\mathrm{mm})$ at $200 \mu \mathrm{g} / \mathrm{mL}$} \\
\cline { 3 - 6 } & & Fusarium solani & Mucor species & Aspergillus flavus & Aspergillus niger \\
1 & $3 \mathrm{a}$ & 17 & 18 & 14 & 10 \\
2 & $3 \mathrm{~b}$ & 6 & 17 & 10 & 12 \\
3 & $3 \mathrm{c}$ & 8 & 10 & 12 & 13 \\
4 & $3 \mathrm{~d}$ & 18 & 11 & 16 & 10 \\
5 & $3 \mathrm{e}$ & 25 & 15 & 18 & 26 \\
6 & $3 \mathrm{f}$ & 15 & 25 & 15 & 28 \\
7 & $3 \mathrm{~g}$ & 25 & 16 & 27 & 15 \\
8 & $3 \mathrm{~h}$ & 5 & 15 & 15 & 10 \\
9 & $3 \mathrm{i}$ & 16 & 18 & 20 & 18 \\
10 & $3 \mathrm{j}$ & 26 & 18 & 20 & 25 \\
11 & Terbinafine & 24 & 22 & 28 & 24 \\
\hline
\end{tabular}

Table III

$\mathrm{IC}_{50}$ values $(\mu \mathrm{g} / \mathrm{mL})$ of DPPH, $\alpha$-amylase and butyrylcholineasterase assay

\begin{tabular}{|c|c|c|c|c|}
\hline \multirow[t]{2}{*}{ SL. No. } & \multirow[t]{2}{*}{ Compound code } & \multicolumn{3}{|c|}{$\mathrm{IC}_{50}$ values $(\mu \mathrm{g} / \mathrm{mL})$} \\
\hline & & DPPH & a-amylase & butyrylcholineasterase \\
\hline 1 & $3 a$ & 12 & 22 & - \\
\hline 2 & $3 b$ & 10 & 25 & 185.8 \\
\hline 3 & $3 c$ & 13 & 12 & - \\
\hline 4 & $3 d$ & 10 & 9 & - \\
\hline 5 & $3 e$ & - & - & - \\
\hline 6 & $3 f$ & 246.3 & 282.1 & 238.8 \\
\hline 7 & $3 g$ & - & 294.2 & - \\
\hline 8 & $3 \mathrm{~h}$ & - & - & - \\
\hline 9 & $3 \mathrm{i}$ & - & 285.1 & 271.4 \\
\hline 10 & $3 \mathbf{j}$ & 151.2 & - & - \\
\hline 11 & Ascorbic acid & 11.9 & - & - \\
\hline 12 & Acarbose & - & 17.1 & - \\
\hline 13 & Galantaminehydrobromide & - & - & 4.6 \\
\hline
\end{tabular}

\section{Butyryl cholinesterase assay}

The synthesized compounds were screened for their inhibition ability against butyryl cholinesterase enzyme. The experiment was performed in triplicates and galantamine hydrobromide was used as a positive control $\left(\mathrm{IC}_{50} 4.6 \mu \mathrm{g} / \mathrm{mL}\right)$. It can be seen from results that three compounds $3 \mathbf{b}$ ( $\left.\mathrm{IC}_{50} 185.8 \mu \mathrm{g} / \mathrm{mL}\right)$, 3f $\left(\mathrm{IC}_{50}\right.$ $238.8 \mu \mathrm{g} / \mathrm{mL}$ ) and $3 \mathbf{i}\left(\mathrm{IC}_{50} 271.4 \mu \mathrm{g} / \mathrm{mL}\right.$ ) were found potential inhibitor of butyrylcholinesterase enzyme while the rest of the compounds were not active against enzyme (Table III).

Table III shows the results of anti-oxidant activity and enzyme inhibition. The compounds were subjected for enzyme inhibition for two different enzymes.

\section{Discussion}

Previously, the reported synthesis of thioureas by conventional method has not delivered excellent yield and tedious reaction work ups were involved and the reaction was completed in 5 hours.

It is well recognized that thioureas possess a wide spectrum of biological activities and previously our research group and other researchers have highlighted the biological application of different acyl and aryl thioureas. To the best our knowledge, there is no paper published related to the octanoyl thiourea. However, Correa et al. (2015) reported the BSA- and DNAbinding studies of thiourea complexes against lung and prostate tumor cells. The antimicrobial activities of thioureas were reported in which few derivatives were found to be good against E. coli (Zhong et al., 2008). Madabhushi et al. (2014) reported the benzimidazole linked chiral thioureas as antibacterial and anti-cancer agents. The biological applications of acyl/aryl thioureas have comprehensively been discussed (Saeed et al., 2014). Tameryn et al. published the antiparasitic 
and cytotoxic activities of polyamide thioureas (Stringer et al., 2013). Saeed et al. reported the coumarin linked thioureas as cholinesterase inhibitors (Saeed et al., 2015). Saeed et al reported amino benzene sulfonamide thiourea conjugates as carbonic anhydrase inhibitors (Saeed et al., 2014). But herein, we have reported the multi-target activities of synthesized thioureas.

But all these other reported work on thioureas have demonstrated their different biological activities, herein, we first time, report the multi-target potential of octanoyl thioureas.

The nature of substrates $\mathrm{H}_{2} \mathrm{NR}$, strongly influence the yield of the final products obtained (Scheme 1). The substituents like $\mathrm{F}, \mathrm{Br}, \mathrm{Cl}$, methyl, $\mathrm{OMe}$, attached at the ortho, meta and para positions on the aromatic ring in $\mathrm{H}_{2} \mathrm{NR}$ moiety show the strong mesomeric effect by releasing electrons through delocalization of lone pairs in spite of the inductive effect (-I) that result in an increase of nucleophilic character of the amino group. It is well established that the resonance effect is stronger than inductive effect and the net result is electron releasing to rest of the molecule. However, the reactivity of di- and higher substituted substrate has been enhanced owing to their increased electron releasing capability and of better yield as the data suggest. This has been reflected in the increase of product yield in the following order; $\mathrm{Br}>$ methyl $>\mathrm{OM}$ $>$ F. The nitro-containing substrates have shown lowest yield due to its electron pulling nature from the aromatic ring, rendering the ring electron deficient, which in turn diminishes the nucleophilic character of amine.

The significant absorptions observed in the FT-IR spectra of all the synthesized substituted thioureas are listed in spectroscopic data along with the respective compounds in the experimental section. The tentative assignments for functionalities are made according to the literature (Saeed et al., 2014). The absence of $v(\mathrm{~S}-\mathrm{H})$ vibration in the range of $2529-2588 \mathrm{~cm}^{-1}$ confirmed the conversion of isothiocyanate moiety into thiourea (NHCSNH-) functionality. The substituted thioureas behave both as a monodentate and bidentate ligands, depending upon the reaction conditions. The characteristic IR bands of substituted thioureas are found around; 3120-3402 (NH), 2960-3090 $\mathrm{Ph}(\mathrm{CH}), 1660-1720$ (C=O), 1540-1620 (CN), 1243-1277 (C=S) and 1130-1185 (C-S) (Saeed et al., 2013). The addition of the amine to the C-N double bond took place by attacking the carbon atom in the isothiocyanate group resulting in the formation of the desired compounds. After complete reaction, the strong band at $2000 \mathrm{~cm}^{-1}$ (N-C-S) in the isothiocyanate disappeared (Saeed et al., 2016). Instead of the expected normal carbonyl (C-O) absorption around $1710 \mathrm{~cm}^{-1}$ a medium strong band at $1665-78 \mathrm{~cm}^{-1}$ suggested a possible hydrogen bond formation between the $\mathrm{H}$-atom of the $\mathrm{NH}$-group and the $\mathrm{O}$-atom of the carbonyl group. The effect and type of hydrogen bonding in thioureas have been excellently discussed by us in earlier reported paper [Saeed et al., 2011]. The ${ }^{1} \mathrm{H}$ NMR data for the synthesized disubstituted thioureas showed that the $\mathrm{NH}$ hydrogen resonates considerably down-field from other resonances in the spectra. The proton chemical shifts were found around 11-12 ppm for free and hydrogen bonded $\mathrm{NH}$, respectively, and aromatic protons appeared downfield between 7.23 and 8.50 ppm in their usual regions. It was also observed in our previous work that the coordinating or highly polar solvents like DMSO- $\mathrm{d}_{6}$ had a profound effect on the free $\mathrm{NH}$ protons chemical shift and appeared more downfield as compared with the non-coordinating solvents like $\mathrm{CDCl}_{3}, \mathrm{C}_{6} \mathrm{D}_{6}$ and $\mathrm{CD}_{2} \mathrm{Cl}_{2}$. This shift could be attributed to the possible hydrogen bonding between the $\mathrm{NH}$ and sulfoxide (S-O) moiety (Saeed et al., 2014). The ${ }^{13} \mathrm{C}$ NMR data explicitly show all the signals due to the distinct carbons present in compounds (3a-i). The aromatic carbon resonances of the thiourea 1 were assigned on the basis of signal intensities and then were compared with the reported values (Saeed et al., 2012). The chemical shifts of the carbons pertaining to CONH and CSNH moieties of the substituted thiourea ligands resonate around 166-68 and 177-80 ppm respectively.

The structure activity relationship was developed to rationalize the results of biological activities. The extensive applications of thiourea in biological field have prompted researchers to explore the structureactivity relationship. Thioureas are capable of showing inter and intra molecular hydrogen bonding which helps in acting as receptors as shown in Figure 1. A series of ten compounds were designed and synthesized to develop structure activity relationship for different five bioassays (antibacterial, antifungal, anti-oxidant and enzyme inhibition ( $\alpha$-amylase and butyl cholinesterase). Compounds 3a-d showed better results in anti-oxidant activity, probably due to the substitution at para-position of the benzene ring. Compounds 3a-c was found potent inhibitors against alpha-amylase enzyme and the series of compounds were found to be less potent against butylcholinesterase enzyme inhibition. In case of antibacterial and antifungal activity, the derivatives 3a-e showed excellent results and $3 c$ derivative showed higher activity than the standard drug. Compounds 3a-e with para-substitution showed better activity than meta and ortho substituted derivatives.

\section{Conclusion}

A series of new 1-octanoyl-3-aroyl was designed and synthesized. The biological assay results indicated that most of the compounds possessed in vitro antifungal activity against fluconazole-resistant Trichophyton 


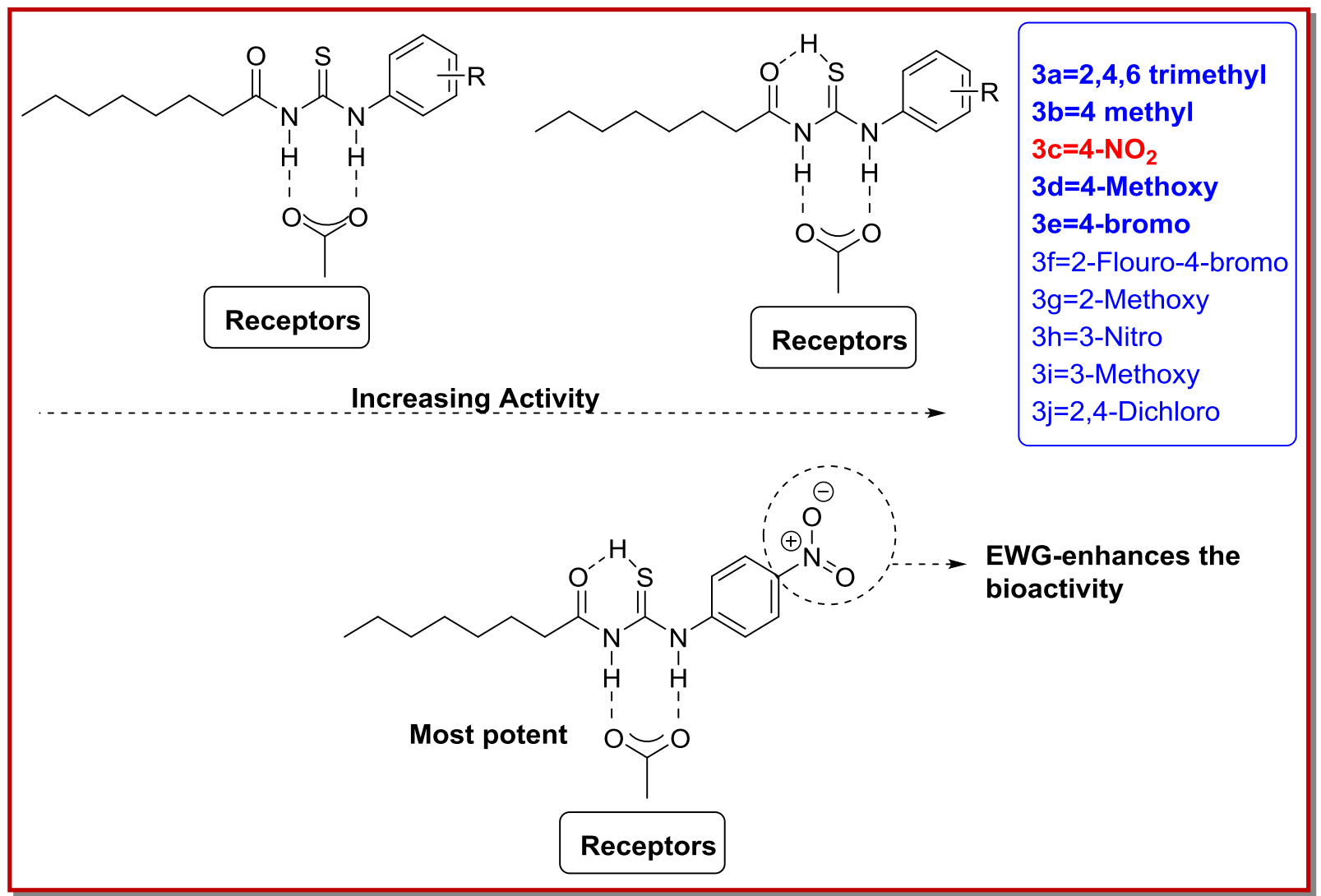

Figure 1: SAR relationship of series of 1-octanoyl-3-aroyl thioureas

rubrum and Cryptococcus neoformans. Compounds (3a-i) showed adequate activity.

\section{Acknowledgement}

We are thankful to Quaid-i-Azam University, Islamabad, Pakistan for providing a healthy environment for research.

\section{Conflict of Interest}

All authors have completed the ICMJE uniform disclosure form and declare no support from any organization for the submitted work.

\section{References}

Babazadeh-Qazijahani M, Badali H, Irannejad H, Afsarian MH, Emami S. Imidazolylchromanones containing non-benzylic oxime ethers: Synthesis and molecular modeling study of new azole antifungals selective against Cryptococcus gattii. Eur J Med Chem. 2014; 76: 264-73.

Bassetti M, Merelli M, Temperoni C, Astilean A. New antibiotics for bad bugs: Where are we? Ann Clin Microbiol Antimicrob. 2013; 12: 12 .

Correa RS, de Oliveira KM, Delolo FG, Alvarez A, Mocelo R, Plutin AM, Cominetti MR, Castellano EE, Batista AA, Ru(II)- based complexes with $\mathrm{N}$-(acyl)-N',N'-(disubstituted)thiourea ligands: Synthesis, characterization, BSA- and DNA-binding studies of new cytotoxic agents against lung and prostate tumour cells. J Inorg Biochem. 2015; 150: 63-71.

Ellman GL, Courtney KD, Andres V jr, Featherstone RM. A new and rapid colorimetric determination of acetylcholinesterase activity. Biochem Pharmacol. 1961; 7: 88-95.

Gilbert MJ, Thornton CR, Wakley GE, Talbot NJ. A p-type ATPase required for rice blast disease and induction of host resistance. Nature 2006; 440: 535-39.

Gorja DR, Mukherjee S, Meda CLT, Deora GS, Kumar, KL, Jain A, Chaudhari GH, Chennubhotla KS, Banote RK, Kulkarni P, Parsa KV, Novel N-indolylmethyl substituted olanzapine derivatives: Their design, synthesis and evaluation as PDE4B inhibitors. Org Biomol Chem. 2013; 11: 2075-79.

Hoagland D, Zhao Y, Lee RE. Advances in drug discovery and development for pediatric tuberculosis. Mini Rev Med Chem. 2016; 16: 481-97.

Jiang $Z$, Gu J, Wang C, Wang $S$, Liu N, Jiang $Y$, Dong G, Wang Y, Liu Y, Yao J, Miao Z, Zhang W, Sheng C. Design, synthesis and antifungal activity of novel triazole derivatives containing substituted 1,2,3-triazole-piperdine side chains. Eur J Med Chem. 2014; 82: 490-97.

Jain KS, Khedkar VM, Arya N, Rane PV, Chaskar PK, Coutinho EC. Design, synthesis and evaluation of condensed 2H-4-arylaminopyrimidines as novel antifungal agents. Eur J Med Chem. 2014; 77: 166-75.

Leroux P. Recent developments in the mode of action of 
fungicides. Pestic Sci. 1996; 47: 191-97.

Madabhushi S, Mallu KKR, Vangipuram VS, Kurva S, Poornachandra Y, Kumar CG. Synthesis of novel benzimidazole functionalized chiral thioureas and evaluation of their antibacterial and anticancer activities. Bioorg Med Chem Lett. 2014; 24: 4822-25.

Mert S, Kasimogullari R, Iça T, Çolak F, Altun A, Ok S. Synthesis, structure-activity relationships, and in vitro antibacterial and antifungal activity evaluations of novel pyrazole carboxylic and dicarboxylic acid derivatives. Eur J Med Chem. 2014; 78: 86-96.

Mohammed A, Abalaka ME. Agrobacterium transformation: A boost to agricultural biotechnology. J Med Gene Geno. 2011; 3: $126-30$

Partida-Martinez LP, Hertweck C. Pathogenic fungus harbours endosymbiotic bacteria for toxin production. Nature 2005; 437: 884-88.

Raza R, Saeed A, Arif M, Mahmood S, Muddassar M, Raza A, Iqbal J. Synthesis and biological evaluation of 3thiazolocoumarinyl schiff-base derivatives as cholinesterase inhibitors. Chem Biol Drug Des. 2012; 80: 605-15.

Russell PE. A century of fungicide evolution. J Agric Sci. 2005; 143: $11-25$

Saeed A, Khurshid A, Bolte M, Fantoni AC, Erben MF. Intraand intermolecular hydrogen bonding and conformation in 1 -acyl thioureas: An experimental and theoretical approach on 1-(2-chlorobenzoyl)thiourea. Spectrochimica Acta Part A: Mol Biomol Spec. 2015; 143: 59-66.

Saeed A, Ashraf S, White JM, Soria DB, Franca CA, Erben MF. Synthesis, X-ray crystal structure, thermal behavior and spectroscopic analysis of 1-(1-naphthoyl)-3-(halo-phenyl)thioureas complemented with quantum chemical calculations. Spectrochimica Acta Part A: Mol Biomol Spec. 2015; 150: 409-18.

Saeed A, Flörke U, Erben MF. A review on the chemistry, coordination, structure and biological properties of 1-(acyl/ aroyl)-3-(substituted) thioureas. J Sulfur Chem. 2014; 45: 31855.

Saeed A, Zaib S, Ashraf S, Iftikhar J, Muddassar M, Zhang KY, Iqbal J. Synthesis, cholinesterase inhibition and molecular modelling studies of coumarin linked thiourea derivatives. Bioorg Chem. 2015; 63: 58-63.

Saeed A, Channar PA, Iqbal Q, Mahar J. C-H Arylation using acyl thiourea ligands: Applications in the synthesis of 3,6diaryl-[1,2,4] triazolo $[3,4-\mathrm{b}][1,3,4]$ thiadiazoles. Chinese Chem Lett. 2016; 27: 37-40.

Saeed A, Ashraf S, White JM, Soria, DB, Franca, CA, Erben, MF. Synthesis, $X$-ray crystal structure, thermal behavior and spectroscopic analysis of 1-(1-naphthoyl)-3-(halo-phenyl)thioureas complemented with quantum chemical calculations. Spectrochimica Acta Part A: Mol Biomol Spec. 2015; 150: 409-18.

Saeed A, Khurshid A, Bolte M, Fantoni AC, Erben MF. Intraand intermolecular hydrogen bonding and conformation in 1 -acyl thioureas: An experimental and theoretical approach on 1-(2-chlorobenzoyl)thiourea. Spectrochimica Acta Part A: Mol Biomol Spec. 2015; 143: 59-66.
Saeed A, Flörke U, Erben MF. The role of substituents in the molecular and crystal structure of 1-(adamantane-1carbonyl)-3-(mono)-and 3, 3-(di) substituted thioureas. J Mol Struc. 2014; 1065: 150-59.

Saeed A, Erben MF, Bolte M. Synthesis, structural and vibrational properties of 1-(adamantane-1-carbonyl)-3-halophenyl thioureas. Spectrochimica Acta Part A: Mol Biomol Spec. 2013; 102: 408-13.

Saeed A, Khurshid A, Jasinski JP, Pozzi CG, Fantoni AC, Erben MF. Competing intramolecular $\mathrm{N} \mathrm{H} \cdots$ O C hydrogen bonds and extended intermolecular network in 1-(4chlorobenzoyl)-3-(2-methyl-4-oxopentan-2-yl)thiourea analyzed by experimental and theoretical methods. Chem Phy. 2014; 431: 39-46.

Saeed A, Erben MF, Flörke U. Effect of fluorine substitution on the crystal structures and vibrational properties of phenylthiourea isomers. J Mol Struc. 2010; 982: 91-99.

Saeed A, Ashraf S, Flörke U, Espinoza ZYD, Erben MF, Pérez H. Supramolecular self-assembly of a coumarine-based acylthiourea synthon directed by $\Pi$-stacking interactions: Crystal structure and Hirshfeld surface analysis. J Mol Struc. 2016; 1111: 76-83.

Saeed A, Shaheen U, Hameed A, Naqvi SH. Synthesis, characterization and antimicrobial activity of some new 1(fluorobenzoyl)-3-(fluorophenyl)thioureas. J Fluorine Chem. 2009; 130: 1028-34.

Saeed A, Erben MF, Abbas N, Flörke U. Synthesis, crystal Xray diffraction structure, vibrational properties and quantum chemical calculations on 1-(4-(4-Fluorobenzamido)phenyl)-3(4-fluorobenzoyl)thiourea. J Mol Struc. 2010; 984: 240-45.

Saeed A, Erben MF, Shaheen U, Flörke U. Synthesis, structural and vibrational properties of 1-(4-Fluorobenzoyl)-3-(isomeric fluorophenyl)thioureas. J Mol Struc. 2011; 1000: 49-57.

Schroeder DC. Thioureas. Chem Rev. 2009; 55: 181-228.

Stringer T, Taylor D, Kock CD, Guzgay H, Au A, An SH, Sanchez B, O'Connor R, Patel N, Land KM, Smith PJ. Synthesis, characterization, antiparasitic and cytotoxic evaluation of thioureas conjugated to polyamine scaffolds. Eur J Med Chem. 2013; 69: 90-98.

Wang L, Zhang YY, Wang L, Liu FY, Cao LL, Yang J, Qiao $\mathrm{C}$, Ye Y. Benzofurazan derivatives as antifungal agents against phytopathogenic fungi. Eur J Med Chem. 2014; 80: $535-42$.

Zaib S, Saeed A, Stolte K, Flörke U, Shahid M, Iqbal J. New aminobenzene sulfonamide-thiourea conjugates: Synthesis and carbonic anhydrase inhibition and docking studies. Eur J Med Chem. 2014; 78: 140-50.

Zhong Z, Xing R, Liu S, Wang L, Cai S and Li P. Synthesis of acyl thiourea derivatives of chitosan and their antimicrobial activities in vitro. Carbohydrate Res. 2008; 343: 566-70.

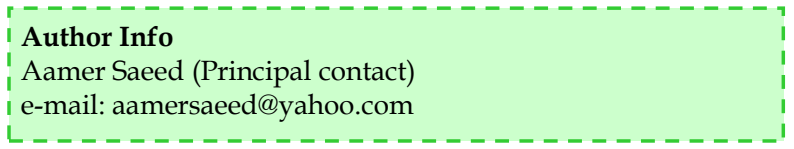




\section{Your feedback about this paper}

1. Number of times you have read this paper 0

2. Number of times you have seen the video clip 0

3. Quality of paper Click

4. Your comments 